\title{
Amiodarone in the treatment of atrial fibrillation of patients with rheumatic heart disease after valve replacement
}

\author{
Kebiao Chen ${ }^{1}$, Li Qin'², \\ Xin $\mathrm{Lu}^{3}$, Tao Xia ${ }^{4}$, Qing $\mathrm{Gu}^{5}$
}

\begin{abstract}
Objectives: To explore the efficacy of amiodarone in the treatment of atrial fibrillation for patients with rheumatic heart disease after valve replacement.

Methods: Eighty-six patients with rheumatic heart disease who were hospitalized between June 2016 and June 2017 and developed atrial fibrillation after valvular heart valve replacement were randomly divided into a control group and an observation group, 42 cases in each group. The control group was treated with routine medical treatment, while the observation group was given amiodarone on the basis of routine treatment. The cardiac function of the two groups were observed and recorded. Postoperative atrial fibrillation conversion rate, sinus rhythm maintenance rate, intensive care unit (ICU) monitoring time and hospital stay were compared between the two groups.

Results: Compared with the control group, the improvement of cardiac function indexes of the observation group was better, and the difference was statistically significant $(P<0.05)$. The atrial fibrillation conversion rate and the maintenance rate of sinus rhythm of the observation group were $76.2 \%$ and $47.6 \%$ respectively, which were significantly higher than $57.1 \%$ and $33.3 \%$ of the control group; the differences had statistical significance $(P<0.05)$. The ICU monitoring time and hospitalization time of the patients in the observation group were $(1.69 \pm 0.91) \mathrm{d}$ and $(10.24 \pm 1.11) \mathrm{d}$ respectively, which were significantly shorter than $(2.83 \pm 0.95)$ $d$ and $(14.07 \pm 1.17) d$ in the control group $(P<0.05)$; the differences were statistically significant $(P<0.05)$. Conclusion: Amiodarone can effectively treat valve replacement associated atrial fibrillation of patients with rheumatic heart disease. It can significantly improve the heart function, prevent the recurrence of atrial fibrillation, maintain sinus rhythm after operation, and shorten the time of ICU monitoring and hospitalization.
\end{abstract}

KEYWORDS: Amiodarone, Rheumatic heart disease, Atrial fibrillation.

doi: https://doi.org/10.12669/pjms.35.4.1298

How to cite this:

Chen K, Qin L, Lu X, Xia T, Gu Q. Amiodarone in the treatment of atrial fibrillation of patients with rheumatic heart disease after valve replacement. Pak J Med Sci. 2019;35(4):918-922. doi: https://doi.org/10.12669/pjms.35.4.1298

This is an Open Access article distributed under the terms of the Creative Commons Attribution License (http://creativecommons.org/licenses/by/3.0), which permits unrestricted use, distribution, and reproduction in any medium, provided the original work is properly cited.

1. Kebiao Chen,

2. Li Qin,

3. Xin Lu,

4. Tao Xia

5. Qing Gu

1-5: Department of Cardiovascular Surgery,

Taian Central Hospital, Shandong, 271000, China.

Correspondence:

Qing Gu,

Department of Cardiovascular Surgery,

Taian Central Hospital,

Shandong, 271000, China.

E-mail: gu223q@163.com

* Received for Publication:

* Revision Received:

* Revision Accepted:
October 24, 2018

April 25, 2019

May 8, 2019

\section{INTRODUCTION}

Atrial fibrillation is the most common persistent arrhythmia in clinical practice. ${ }^{1,2}$ The incidence of atrial fibrillation after valve replacement is as high as $65 \%$ among patients with rheumatic heart disease. The incidence of atrial fibrillation after simple mitral valve replacement and aortic valve replacement is $35.1 \%$ and $36.2 \%$ respectively, and the incidence of atrial fibrillation after mitral valve replacement combined with aortic valve replacement can be as high as $55.4 \% .{ }^{3}$ In addition, postoperative atrial fibrillation generally has a low conversion rate, and the sinus rhythm is poor after 
conversion, which may have adverse effects on patients, cause hemodynamic disorders and even cardiogenic shock, and increase risks of stroke, heart failure and hospitalization mortality. ${ }^{4,5}$ Therefore, how to maintain sinus rhythm effectively after valve replacement, prevent recurrence of atrial fibrillation, avoid complications such as stroke and heart failure, and effectively improve the quality of life of patients have become important problems in cardio-thoracic surgery.

A recent study shows that amiodarone has a unique role in preventing postoperative atrial fibrillation. ${ }^{6}$ Roy D et al. found that amiodarone was safe and effective in the conversion and maintenance of sinus rhythm and can reverse atrial fibrillation by extending atrial effective refractory period and non-competitive antiepinephrine effect. ${ }^{7}$ Amiodarone has been widely used in the conversion of atrial fibrillation, and its conversion efficiency is as high as $55 \% .{ }^{8}$ This study investigated the effect of amiodarone on atrial fibrillation of patients with valvular replacement associated rheumatic heart disease, aiming to provide a reference for the treatment of valve replacement associated atrial fibrillation.

\section{METHODS}

Research Data: In the study, eight-four patients with rheumatic heart disease who were admitted into our hospital between June 2016 and June 2017 and had valvular replacement associated rheumatic heart disease were selected. Patients who underwent elective valvular replacement, had rheumatic heart disease in combination with continuous atrial fibrillation, had no other types of arhythmia, had cardiac function no higher than grade III, ${ }^{9}$ satisfied the application indication of amiodarone, had normal level of electrolyte and acidity and alkalinity, and had heart rate lower than 70 times/ min were included. The patients were divided into a control group and an observation group according to the random digital table method, 42 cases each group. The gender composition, average age, left atrial diameter (LAD), duration of atrial fibrillation, heart function classification of (New York Heart
Table-I: Baseline characteristics of study population.

\begin{tabular}{lcc}
\hline Group & Observation group & Control group \\
\hline Male/female & $22 / 20$ & $21 / 21$ \\
Age (year) & $65.36 \pm 10.77$ & $65.45 \pm 10.82$ \\
Duration of atrial & $30.96 \pm 14.93$ & $31.06 \pm 15.02$ \\
$\quad$ fibrillation (month) & & \\
LAD (mm) & $45.87 \pm 3.95$ & $47.06 \pm 3.35$ \\
LVEF(\%) & $46.7 \pm 4.32$ & $47.5 \pm 4.03$ \\
Cardio-thoracic ratio & $0.54 \pm 0.07$ & $0.56 \pm 0.08$ \\
NYHA & $2.50 \pm 0.51$ & $2.58 \pm 0.50$ \\
\hline
\end{tabular}

Association (NYHA), cardio-thoracic ratio and left ventricular ejection fraction (LVEF) of the two groups had no remarkable differences ( $\mathrm{P}>0.05)$; hence the results were comparable (Table-I). This study was approved by the ethics committee of our hospital. All the patients signed informed consent.

Method: Patients in the control group received routine postoperative drug treatment including oral administration of drugs for diuresis, anticoagulation and routine application of antibiotics. On the basis of routine treatment, the observation group was additionally treated with amiodarone (Sanofi Minsheng Pharmaceutical Co., Ltd.; batch number: 7h90042). On the day of the operation, a micro infusion pump was used to pump $600 \mathrm{mg}$ of amiodarone injection at a speed of $50 \mathrm{mg} / \mathrm{h}$, for 12 hours. On the postoperative $1^{\text {st }}$ day, the patients orally took amiodarone after recovery of diet, 3 times a day; one week later, the patients took it twice each day; one week later, the patients took it once daily. The course of treatment was one month.

Observational Indexes: Cardiac function indexes including peak velocity of peak A (VA), dispersion of wave $\mathrm{P}(\mathrm{Pd})$, the maximum time limit of wave $P$ (Pmax), the maximum volume of the left atrium $\left(\mathrm{LAV}_{\max }\right)$ and ventricular rate were observed after treatment.

The conversion rate of atrial fibrillation, maintenance rate of sinus rhythm, ICU monitoring time and hospitalization time of the two groups were also observed after treatment.

Statistical Processing: Data were analyzed using SPSS 21.0. Measurement data were expressed

Table-II: Comparison of different cardiac function indexes (mean \pm SD).

\begin{tabular}{lcccc}
\hline Group & Observation group & Control group & $t$ & $P$ \\
\hline VA(cm/s) & $63.41 \pm 6.65$ & $58.28 \pm 6.76$ & 3.812 & $<0.05$ \\
Pd(ms) & $45.48 \pm 6.09$ & $50.02 \pm 6.24$ & 3.701 & $<0.05$ \\
Pmax $(\mathrm{ms})$ & $112.03 \pm 11.26$ & $121.51 \pm 10.99$ & 4.278 & $<0.05$ \\
LAVmax $(\mathrm{cm} 3)$ & $19.10 \pm 5.34$ & $26.38 \pm 5.61$ & 6.635 & $<0.05$ \\
Heart rate(times/mi) & $80.41 \pm 3.37$ & $88.89 \pm 3.63$ & 12.124 & $<0.05$ \\
\hline
\end{tabular}


as mean \pm standard deviation (SD), Comparison between groups was performed using $t$ test. Enumeration data were processed using Chi-square test. Difference was thought statistically significant if the value of $p$ was smaller than 0.05 .

\section{RESULTS}

Cardiac Function of the Two Groups: The improvement of the cardiac function indexes of the observation group was better than that in the control group after treatment, and the difference was statistically significant $(\mathrm{P}<0.05$, Table-II).

The conversion of atrial fibrillation and sinus rhythm of the two groups: After treatment, the maintenance rate of sinus rhythm and conversion rate of atrial fibrillation were obviously superior to those of the control group, and the differences between the two groups were statistically significant $(\mathrm{P}<0.05$, Table-III).

ICU monitoring time and hospitalization time of the two groups: After treatment, the ICU monitoring time of the observation group was significantly shorter than that of the control group $((1.69 \pm 0.91) \mathrm{d}$ vs. $(2.83 \pm 0.95) \mathrm{d})$, and the difference was statistically significant $(\mathrm{t}=3.841, \mathrm{P}<0.05)$. The hospitalization time of the observation group was significantly shorter than that of the control group $((10.24 \pm 1.11) \mathrm{d}$ vs. $14.07 \pm 1.17) \mathrm{d}$, and the difference was statistically significant $(t=7.254$, $\mathrm{P}<0.05$, Table-IV).

\section{DISCUSSION}

Rheumatic heart disease is induced by rheumatic fever which involves heart valve. With the improvement of living standard, the incidence of rheumatic heart disease is increasing. ${ }^{10}$ Valve replacement has become the main therapy for rheumatic heart disease. ${ }^{11}$ Patients often have atrial fibrillation after undergoing rheumatic heart valve replacement. Patients with mild atrial fibrillation may have disturbance of hemodynamics in the perioperative period, and patients with severe atrial fibrillation may have severe heart failure, stroke, and cardiogenic shock or even die. Studies found that improving the conversion rate of atrial fibrillation and maintaining sinus rhythm was the key after rheumatic heart disease associated valve replacement. ${ }^{12,13}$

Amiodarone which is the third class of antiarrhythmic drug is a $\beta$ and $\alpha$ adrenergic receptor inhibitor. Its bioavailability is about $50 \%$. It is mainly distributed in the organs which were rich in fat, such as lymph gland, liver, kidney and lung. $34 \%$ of amiodarone in plasma can be combined with beta lipoprotein, and $62 \%$ can be combined with albumin; moreover it can be eliminated through metabolism in the lung. The half-life of the drug is longer, $15 \mathrm{~d}$ to $28 \mathrm{~d}$. It can be absorbed by tissue after $4.6 \mathrm{~h}$ when the dose was $0.8 \mathrm{~g}$. The blood concentration of amiodarone can reach the peak at the $4^{\text {th }}$ to $6^{\text {th }} \mathrm{h}$, and a low blood concentration can still be detected even half a year after withdrawal. ${ }^{14,15}$ Its main pharmacological function is to eliminate re-entrant excitation, inhibit the rapid sodium ion internal flow of the atrial and myocardial conduction fibers and reducing sinus-node selfactivity through prolonging the action potential and effective refractory period of the myocardial tissue of patients. ${ }^{16}$ In addition, amiodarone has four kinds of antiarrhythmic functions. It can inhibit the potassium channel and has no influence on the height of resting membrane potential and action potential. Although there is a slight negative inotropic effect in static injection, it usually will not inhibit the left ventricular function. Moreover it has direct dilation effect on the coronary artery and the surrounding blood vessels, which is beneficial to improve the postoperative haemodynamics of patients. ${ }^{17,18}$ Compared with other antiarrhythmic drugs, amiodarone has higher conversion rate and better sinus rhythm maintenance. ${ }^{19}$ In this study, the conversion rate of atrial fibrillation and maintenance rate of sinus rhythm of the observation group

Table-III: The conversion of atrial fibrillation and maintenance of sinus rhythm $[\mathrm{n}(\%)]$.

\begin{tabular}{lcccc}
\hline Group & Observation group & Control group & $X^{2}$ & $P$ \\
\hline Conversion rate of atrial fibrillation & $32(76.2)$ & $24(57.1)$ & 4.483 & $<0.05$ \\
Maintenance rate of sinus rhythm & $20(47.6)$ & $14(33.3)$ & 4.229 & $<0.05$ \\
\hline
\end{tabular}

Table-IV: The comparison of ICU monitoring time and hospitalization time (mean \pm SD).

\begin{tabular}{lcccc}
\hline Group & Observation group & Control group & $t$ & $P$ \\
\hline ICU monitoring time(d) & $1.69 \pm 0.91$ & $2.83 \pm 0.95$ & 3.841 & $<0.05$ \\
Hospitalization time(d) & $10.24 \pm 1.13$ & $14.07 \pm 1.17$ & 7.254 & $<0.05$ \\
\hline
\end{tabular}


were $76.2 \%$ and $47.6 \%$ respectively, which were significantly higher than those of the control group, and the results were similar to the previous research results. $^{20,21}$ In addition, rate of administration ways of amiodarone can produce different effects. Intravenous administration makes amiodarone take effect late, while oral administration is the opposite. The dosage regimen can be formulated according to the postoperative condition of patients. Amiodarone has few side effects and is less likely to induce complications such as embolism after cardioversion, which is beneficial to the recovery of patients. In this study, the ICU monitoring time and time of hospitalization of the observation group was $(1.69 \pm 0.91) \mathrm{d}$ and $(10.24 \pm 1.13) \mathrm{d}$ respectively, which were significantly shorter than that of the control group, which was similar to the research results of Zhang et al. ${ }^{22}$

In addition, amiodarone can also reduce peripheral vascular resistance and increase myocardial blood pumping, which not only maintain the volume of blood transfusion, but also reduce heart rate. Amiodarone can maintain the oxygen consumption of the myocardium, improve the function of diastole and contraction of the myocardium through expanding the coronary artery, increasing the blood supply of the myocardium and improve the working state of the heart. ${ }^{23}$ Wang et al. found that amiodarone could prolong the interval between PR and QT and improve the structure and function of the myocardium on the basis of lowering blood pressure and slowing down the heart rate. ${ }^{24}$ Jie et al. also found that amiodarone could significantly improve the cardiac recovery of patients with atrial fibrillation. ${ }^{25}$ The results of this study also showed that the indexes of cardiac function in the observation group improved significantly, which was similar to the above research result.

\section{CONCLUSION}

For patients with rheumatic heart disease who developed valve replacement associated atrial fibrillation, amiodarone can improve the cardiac function, prevent the recurrence of atrial fibrillation, maintain sinus rhythm for a long time and accelerate recovery. Although amiodarone is effective in the treatment of postoperative atrial fibrillation of patients with rheumatic heart disease, further studies are still needed. For example, whether use of amiodarone at a smaller dosage or earlier use of amiodarone can achieve better results and how sinus rhythm will change after conversion during long-time follow up remains to be verified through longer clinical follow up.

Declaration of interest: All authors declared there was no conflict interests involved.

\section{Grant Support \& Financial Disclosures: None.}

\section{REFERENCES}

1. Ji Q, Mei Y, Wang X, Feng J, Wusha D, Cai J, et al Combination of irbesartan and amiodarone to maintain sinus rhythm in patients with persistent atrial fibrillation after rheumatic valve replacement. Circ J. 2010;74(9):18731879. doi: 10.1253 / circj.cj-10-0254

2. Mitrić G, Udy A, Bandeshe H, ClementP, Boots R. Variable use of amiodarone is associated with a greater risk of recurrence of atrial fibrillation in the critically ill. Crit Care. 2016;20(1):1-9. doi: 10.1186/s13054-016-1252-2

3. Mou CF, Sun J, Wan YH. Effect of amiodarone on atrial fibrillation after valve replacement for rheumatic heart disease. Guide China Med. 2017;(19):68.

4. Wu G, Huang $\mathrm{CX}$, Tang $\mathrm{YH}$, Jiang $\mathrm{H}$, Wan J, Chen $\mathrm{H}$, et al. Changes of IK, ATP current density and allosteric modulation during chronic atrial fibrillation. Chin Med J (Engl). 2005;118(14):1161-1166.

5. Innes C, Ranaweera A. Amiodarone prevents recurrence of atrial fibrillation. Inpharm Weekly. 2000;1232(1):19-18. doi: 10.2165/00128413-200012320-00043

6. Chen HD, Xie YM, Wang LX, Wu JB. Systematic review of efficacy and safety of Shenmai injection for chronic heart failure. China J Chin Mate Med. 2014;39(18):3650-3611.

7. Roy D, Talajic M, Dorian P, Connolly S, Eisenberg MJ, Green $\mathrm{M}$, et al. Amiodarone to prevent recurrence of atrial fibrillation canadian trial of atrial fibrillation investigators. N Engl J Med. 2000;342(13):913-920. doi: 10.1056/ NEJM200008243430812

8. Kreiss Y, Sidi Y, Gur H. Efficacy and safety of intravenous amiodarone in recent-onset atrial fibrillation: Experience in patients admitted to a general internal medicine department. Postgrad Med J. 1999;75(883):278-281.

9. Jiao $\mathrm{H}$, Wang $\mathrm{XL}$, Chen YJ, Xiang LH, Zhang SN Effects of Shenmai injection on afterdepolarization and triggered activities in left ventricular papillary muscle in rat cardiac: Hypertrophy. China J Chin Mate Med. 2014;39(15):2956-2959.

10. Ding $\mathrm{P}$, Li L, Zhang H, Chen LJ, Deng XJ, Yuan YQ. Comparison of amiodarone plus irbesartan regimen versus amiodarone alone on maintaining sinus rhythm in rheumatic heart disease patients with persistent atrial fibrillation post valve replacement and cordioversion. Chin J Cardiol. 2009;37(6):505-508. doi: 10.3760/cma.j.is sn.0253-3758.2009.06.006.

11. Akhtar RP, Abid AR, Naqshband MS, Mohydin BS, Khan JS. Outcome of double vs. single valve replacement for rheumatic heart disease. J Coll Physicians Surg Pak. 2011;21(1):9-14. doi: 01.2011/JCPSP.0914.

12. Finnerty CC, Mabvuure NT, Ali A, Kozar RA, Herndon DN. The surgically induced stress response. J Parenter Enteral Nutr. 2013;37(5 Suppl):21S-29S. doi: $10.1177 / 0148607113496117$.

13. Chauvin $M$, Shah DC, Haissaguerre $M$, Marcellin $\mathrm{L}$, Brechenmacher $\mathrm{C}$. The anatomic basis of connections between the coronary sinus musculatute and the left atrium in humans. Circulation. 2000;101:647-652. 
14. Davis EM, Packard KA, Hilleman DE. Prophylaxis of postoperative atrial fibrillation in patients undergoing cardiac surgery: Beyond beta-blockers. Pharma 2010;30(7):749,274e-318e. doi: 10.1592/phco.30.7.749.

15. Yang MH. Clinical observation of treatment of 64 cases of tachyarrhythmia with amiodarone. Guide China Med. 2015;13(14):160-161.

16. Xie ZC. Clinical analysis of amiodarone in the treatment of acute myocardial infarction associated ventricular arrhythmias. World Clin Med. 2016;10(20):98.

17. Looi KL, Tang A, Agarwal S. Ventricular arrhythmia storm in the era of implantable cardioverter defibrillator. Postgra Med J. 2015;1079(91):519-526. doi: 10.1136/ postgradmedj-2015-133550.

18. Flaker G, Lopes R D, Hylek E, Wojdyla DM, Thomas L, Al-Khatib SM, et al. Amiodarone, anticoagulation, andclinicalevents in patients with atria lfibrillation: insights from the ARISTOTLE trial. J Am Coll Cardiol 2014;64(15):1541-1550. doi: 10.1016/j.jacc.2014.07.967.

19. Haghjoo M. Pharmacollogical and nonpharmacologicall prevention of atrial fibrillation after coronary artery bypass surgery. J Tehran Heart Cent. 2012;7(1):2-9.

20. Selvaraj T, Kiran U, Das S, Chauhan S, Sahu B, Gharde P. Effect of single intraoperative dose of amiodarone in patients with rheumatic valvular heart disease and atrial fibrillation undergoing valve replacement surgery. Ann Card Anaesth. 2009;12(1):10. doi: 10.4103/0971-9784.42881

21. Kar SK, Dasgupta CS, Goswami A. Effect of prophylactic amiodarone in patients with rheumatic valve disease undergoing valve replacement surgery. Ann Card Anaesth. 2011;14(3):176-182. doi: 10.4103/09719784.83986
22. Zhang RY, Liang CZ, Song YY, Xiang KF. Study on amiodarone to treat atrial fibrillation after cardiac valve replacement in rheumatic heart disease patients. Ningxia Med J. 2011;33(4):304-306. doi: 10.3969/j.issn.10015949.2011.04.006.

23. Mitchell LB, CCS Atrial Fibrillation Guidelines Committee. Canadian cardiovascular society atrial fibrillation guidelines 2010: prevention and treatment of atrial fibrillation following cardiac surgery. Can J Cardiol. 2011;27(1):91-97. doi: 10.1016/j.cjca.2010.11.005.

24. Wang L, Huang XE, Cao J. Clinical study on safety of cantharid in sodium and shenmai injection combined with chemotherapy in treating patients with breast cancer post-operatively. APJCP. 2014;15(14):5597-5600.

25. Jie $\mathrm{S}, \mathrm{Yu} \mathrm{C}, \mathrm{Lu} \mathrm{XH}$, Qian $\mathrm{YN}$, Ding ZN, Tang $X Y$. The effect of amiodarone on cardiac rhythm after cardiopulmonary bypass in patients with atrial fibrillation. J Heart Valve Dis. 2010;19(6):786-788. doi: 10.1083/nrcardio.2010.68-c2.

\section{Authors' Contribution:}

KBC \& LQ: Study design, data collection and analysis.

KBC, XL \& TX: Manuscript preparation, drafting and revising.

KBC \& QG: Review and final approval of manuscript. 